\title{
Research on the Stability of Eco-Industry Chains
}

\author{
Jingfu Guo \& Wenzi Cui \\ School of Economy \& Management, Dalian Nationalities University, Dalian 116600, China \\ E-mail: drguo@dlnu.edu.cn
}

This paper is sponsored by Social Science Research Program of the Education Ministry (08JA850002)

This paper is sponsored by the Fundamental Research Funds for the Central Universities (DC10030206)

This paper is sponsored by Liaoning Social Science Planning Program (L09BJL014)

\begin{abstract}
Eco-industrial chains construct economic activities into the "resource-product-renewable resource" feedback processes based on ecology laws, improve resource utilization, reduce emissions, and improve the quality of economic activities and environmental benefits. Trust and the good cooperation relationships between the enterprises from eco-industrial chains are the prerequisite and basis for running ecological industry systems. A trust mechanism between enterprises, an incentive and punishment system, and a legal system and industrial policies should be established to promote industrial ecology. Government should play a good role of "guidance" and "coordination". There is a smooth linkage between the upstream and downstream of the industrial ecology chain so that "upstream supplies and downstream receives", the stability of the industrial ecology system can be achieved.
\end{abstract}

Keywords: Eco-Industry Chain, Circular economy, Industry policy

\section{Introduction}

Since the beginning of the twentieth century, the world economy has made a rapid growth driven by technology - humans over the past 100 years have created more material wealth than the historical summation before. However, this kind of rapid economic growth is based on the" high investment, high consumption, and high emissions" characteristics. It results in resource depletion, pollution, the deterioration of the ecosystem. Industrial civilization pushes the progress of science and technology and material prosperity. At the same time, it also brings a lot of questions, to a certain extent, it has expressed as counter to human and natural. Therefore, human beings have to re-think and re-examine the modernization of production, life style and values, and even seek new ways of development. The applications of circular economy and industrial ecological theory could make the economic development with high-efficiency, Low-pollution of the environment, resources and energy recycling, and sustainable growth.

We hope to develop economy, create and enjoy the industrial civilization, while minimizing the negative impact on the environment, thus realizing benefits lasting growth and harmony between human and nature as the ideal destination. Under this goal, economists and industry engineers have done a lot of exploration on industry development models. In 1988, Robert U. Ayres proposed the concept of industrial metabolism, carried on the research of human industrial system and natural environment between the material and energy flows. He believes that the economic system is not isolated, but an embedded open ecological systems, which has linkage with natural ecosystems through the material and energy flow. In 1990, United States National Academy of Sciences and the Bell Laboratories jointly organized the first "industrial ecology" Forum. At the Forum, they overviewed industrial ecology concepts, contents, methods and application, and formed the conceptual framework of industrial ecology. The framework describes an industrial bionic system to maintain a sustainable development of the industrial system through imitating ecological systems and structures. In the system, the waste (output) of an industrial activity is the raw materials (inputs) of another industrial activity. Scholars agree that this "material-loop" that can radically conserve the natural resources, reduce waste, and improve the ecological and economic efficiency. Schwarz and Steininger (1995) believe that "in most cases, the waste of a production process of cannot be used in the same production process again, but only be used by different production process". They have raised the importance of mutual cooperation between enterprises to establish an "industrial recycling network".

In 1898, Frosch and Gallopoulos published a paper named "Strategies for Manufacturing", in the paper, they proposed concept of Industrial Ecology, and they pointed that "traditional industrial activity patterns ... should be 
transmitted into to a more complete model: industry ecosystem. In this system, energy and material consumption is optimized, waste discharge is minimized, and the waste of a production process becomes ... the raw materials of another production process". The eco-industrial park is the most successful application of industrial ecological theory. An eco-industrial park is made of manufacture and service enterprises. Through the cooperation among the basic elements of the environment and resources such as energy, water, and raw materials, the park achieves both environmental and economic optimization and harmonious development, ultimately makes the enterprise communities achieve much more overall benefits than the sum of each company's individual benefits.

The stability of the eco-industry chain will have a direct impact on the industry ecosystem stability and sustainability. There are a variety of factors can affect the stability of the industry chain, such as node enterprise personnel changes, the past business process restructuring, and cutting off the old upstream and the downstream businesses' "waste - product" circular flow channels. Enterprise technology change, external environmental impact, organizational changes such as mergers and take-over will enables the entire cooperation system withstand impact, it is even possible that industry ecosystem collapse. Thus, for Ecology industry chain or ecological study on the stability of the network is essential. This paper first outlines the concepts of ecology industry chain, as well as risk factors and major types of eco-industrial chain, and then it does the research on the strategies to improve stability of the Eco-industry chains.

\section{The Type of Ecology Industry Chain}

Ecology industry chain generally refers to a new industrial symbiosis of the circulatory system based on the ecological principle and 3R (Reduce, Reuse and Recycle) principles of circular economy. In this system, enterprise A's waste is the raw material of enterprise B, and enterprise B's waste is the raw material of enterprise $\mathrm{C}, \ldots$, and so on. The enterprises utilize waste in the different phases of the production process and the waste in the production process is recycled. This substance transfer routes is the eco-industry chain, different industry chains comprise industry ecosystem, to achieve industrial ecosystem of metabolic functions. Ecology industry chain is defined as (Zhang 2006): The special chain of resource utilization in the industrial production of metabolic process. The chain connects different industries through the excreta from industrial production and reuses the residues, thus improves the economic value of such substances, which makes the remaining material processing approach as the truly industrial food chain.

In General, eco-industrial chain is made up of enterprises in a region to imitate natural ecosystems' producers, consumers, and decomposers. Under the link of utilizing waste resources, it forms the industry alliance cohesion to realize the ultimate circular utilization of resources and energy in the region. In this article, we provide the following definition for the ecology industry chain system: The industrial ecological system built in an eco-industrial park, understanding the reality of regional economic development, having a well-designed development plan, referring and using natural law, linking waste and by-products, realizing the ultimate circulation of resources and energy in the regional context, to achieve environmental benefit and social benefit maximization. Ecology industry chain link form refers to the inter-linking among the upstream and downstream enterprise of the industry chain, mainly in the following four types: one-to-one, one-to-many and many-to-one, and mixed linkages (See the following Figure).

There is a certain degree of risk in any eco-industrial symbiosis networks. Any elements (materials, energy, information, etc.) of the enterprise in the cooperative network are constrained by relevant members of the EIC and many environmental factors might bring risks to the ecological stability of symbiosis networks. First, each enterprise production scale is not designed to meet the needs of downstream enterprises for its by-product, but the needs of its own products to market. However, if the enterprise's production scale is dependent on upstream enterprise by-products of supply capacity, and there is a certain amount of inevitable "gap" between supply and demand among the upstream and downstream firms (Zhang and Jiang 2008). This would affect the industrial chain of harmony and stability. Second, all the enterprise nodes of the industrial chain have the professional technical requirements on quality of raw material characteristics. Once a node enterprise in the production process or the use of raw materials has some minor changes, the entire supply of eco-industrial chain has a serious impact. Third, members in the industrial symbiosis network might be opportunistic in the process of pursuing their own interests. The asymmetry of information between enterprises could lead to potential degeneration and other operational risks,

\section{Stability Strategy for Ecology Industry Chain}

\subsection{Establishing and Improving the Legal System and Industrial Policies to Promote Industrial Ecology}

First, it is important to establish the laws and regulations to promote eco-industrial system. More specifically, we should card and upgrade the existing environmental laws and regulations, completely eliminate the obstacles 
from industry eco-cooperation in the traditional regulations and practices, encourage cooperation between enterprises, and construct ecological industry chain to play the protection and guidance role in the industrial ecological process. For example, the laws for resources and environment protection, single ecological industry, eco-industry link, etc. The second is the establishment and improvement of relevant industrial policy. Through the development-related fiscal policy, investment policy, financial policy, and market regulation, the government encourages enterprises to adopt cleaner production technology, foster eco-enterprise community progressively, form industrial symbiosis networks gradually, and promote regional recycling economic development. For example, through fiscal and financial support, the government provides the eco-cooperation enterprise tax incentives and financial subsidies. In a given period of time, the ecological enterprises can be reduced or will be exempted from sales tax and delay the income tax to newly developed green products, etc.

3.2 Construct Information sharing platform and Coordinate the Consistency between the Upstream and Downstream Firms

Build a regional enterprise resource information exchange platform to form an exchange mode with dynamic nature, networking, management, services, and customers all in the platform. Collect more comprehensive information on waste stream from all users, establish a fast waste recycling network chain for waste, materials, and products, and make the wastes resources to be obtained, , configured, and exchanged across the enterprises.

The above main approach of industrial ecology is to utilize the upstream businesses waste as raw materials and energy for the downstream enterprise, but that does not mean that the upstream businesses can produce what wastes or how much they want to. On the contrary, the first thing we should remember is to reduce the waste of the upstream businesses in the "food chain" of symbiosis networks, especially the hazardous substances. In other words, each ring of the system cuts resources and considers how much resource the entire symbiosis network need, comparing with the emissions acceptance capacity of symbiosis network. Otherwise, the "food chain" of the symbiosis network will be out of control due to an imbalance at any ring of the network.

\subsection{Government Coordination and Maintenance Functions}

Local government as a "third party" coordinating role for the stability of the network is also extremely important. Because of the special position of the government, it has the independence and authority in coordinating and maintaining the security and stability for the industrial symbiosis network, which will be good for the coexistence of a variety of governance structure, preserving the interests of honest businesses, and maintaining impartiality. In the ecological chain network operation process, local governments may, in accordance with the actual development situation of the industrial symbiosis network, encourage enterprises to exchange by-products, improve the efficiency of resource use, and make enterprises share the benefits of symbiosis. When the relationships between enterprises affect the network stability, the Government, as the Park's management, is best suited to play the role of the "facilitator" to help enterprises realize that "upstream supplies, downstream receives". Through the government's facilitation, the possibility of breaking relationships between enterprises due to small conflicts can be reduced, therefore avoiding a greater loss. In addition, the government has a deterrent role on some enterprise bad behaviors such as degeneration or speculation, maintaining the industry ecosystem stability.

\subsection{Establish Mechanisms for Fostering Trust between Enterprises and Nurture Good Corporate Culture}

Efforts should be made to educate managers with ecology, business philosophy, business ethics, core values, corporate social responsibility, and the ways of cooperation of industrial ecology. If enterprises in the same industrial chain have similar organizational culture, they have a solid foundation to achieve a good communication and friendly cooperation. Especially when the managers share the similar business philosophy and values, they would more likely to establish trust, keep promises and long-term cooperation between enterprises, and maintain the stability of the eco-industry chain. Industrial symbiosis networks have well established trust mechanism. Due to the geographical closeness, good relationships among members of the enterprises would increase the level of trust in the communication.

Therefore, enterprises in the industry ecosystems should strive to promote and integrate their corporate cultures. They should learn and tolerate from each other, communicate and share with each other, and promote the industry ecosystem stability and development.

\section{References}

Frosch RA, \& Gallopoulos NE. (1989). Strategies for manufacturing. Waste from one industrial process can serve as the raw materials for another--thereby reducing the impact of industry on the environment. Sci Am 261(9):94-102. 
Lowe E. (1997). Creating by-product resource exchange: strategies for eco-industrial Parks. Cleaner Product, 5(1-2):58.

SCHWARZ, E. K. STEININGER. (1995). The Industrial Recycling-Network Enhancing Regional Development. Research Memorandum, (4):96-100.

Wang, Zhaohua \& Yin, Jianhua. (2005). Research on running model of industry co-exist net-work in Eco-industrial parks. China soft science, (2):80-85.

Wilderer PA. (2007b). Sustainable water resources management: the science behind the scene. Sustain Sci, 2:1-4.

$\mathrm{Xu}$, Dawei \& Wan, Ziyan. (2005). The comparison analysis of enterprises linkage in eco-industry chains. Economy of Industry Technology, 24(1): 63-66.

Zhang, Yan. (2006). Study on construction and stability of industry eco-system in Eco-industrial parks. Doctoral dissertation of Huazhong Science and Technology Univ., 2006.

\section{Waste material}
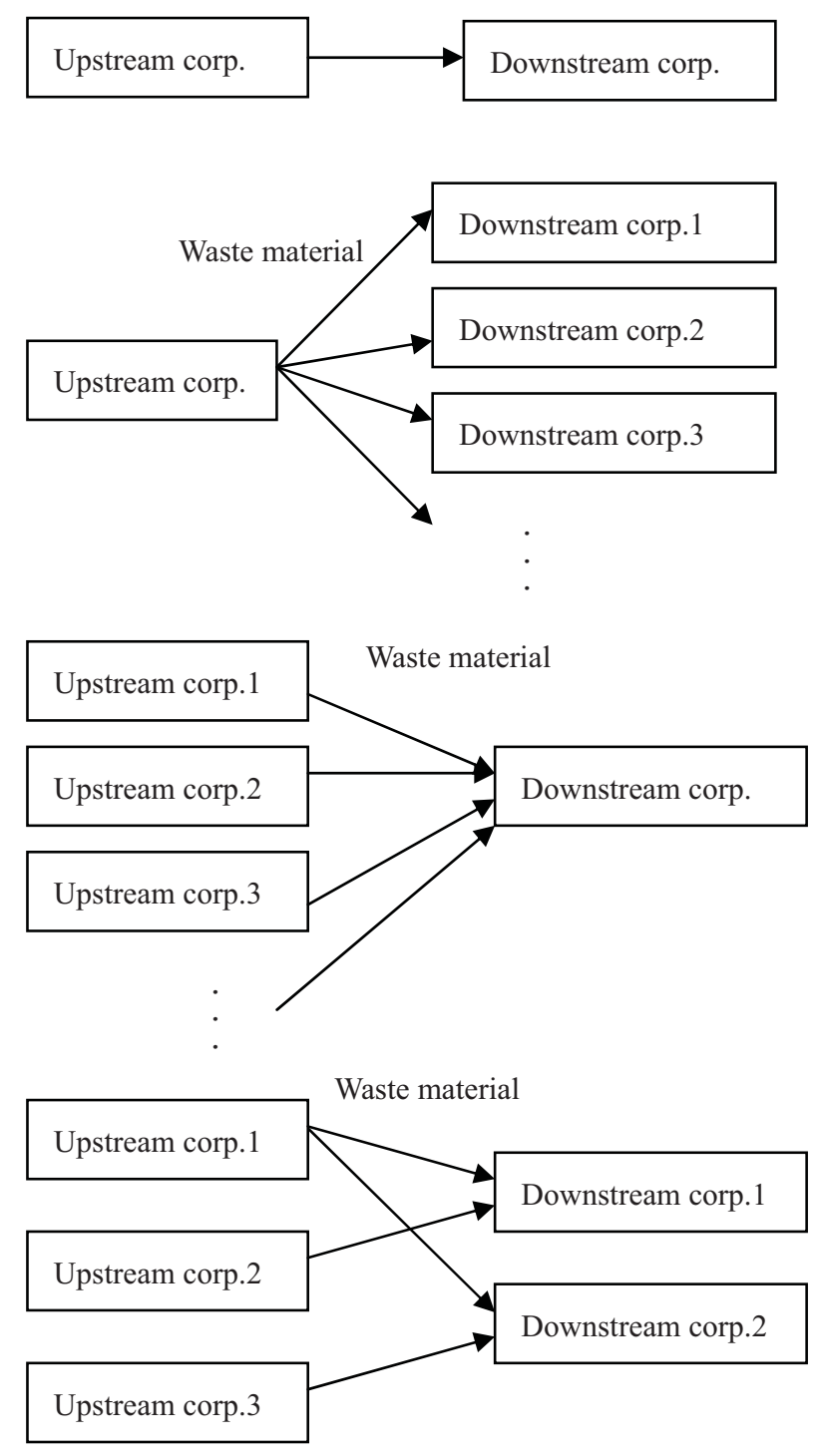

Figure 1. The inter-linking forms of 